\title{
Vorwort der Reihenherausgeber
}

Das Wissen über das Alter oder das Altern und die damit einhergehenden Veränderungen, beispielsweise des Körpers, der Funktionsweisen seiner Organsysteme und der geistigen, seelischen und sozialen Fähigkeiten alt gewordener Menschen nimmt permanent zu. Hier den Überblick zu behalten, ist aufgrund der wachsenden Zahl beteiligter Wissenschaften nicht einfach. Zudem vergeht i. d. R. erhebliche Zeit, bis Wissen mit Anwendungsbezug verfügbar ist und tatsächlich im Alltag seinen Niederschlag findet. Dies gilt auch für Inhalte mit Bezug zur medizinischen, therapeutischen oder pflegerischen Versorgungspraxis für alte Menschen. Gewalt in der Gesundheitsversorgung oder generell gegen ältere Menschen ist zudem ein noch immer tabuisiertes und schambesetztes Thema, ein Tatbestand der Verletzung unserer Menschenrechte, mit gravierenden Folgen für Betroffene, für Einrichtungen des Gesundheitswesens und für eine immer älter werdende Bevölkerung.

In der Buchreihe „Praxiswissen Gerontologie und Geriatrie kompakt“ werden seit nunmehr sechs Jahren und in bereits neun veröffentlichten Bänden Themen und aktuelle Wissensbestände dargelegt, die für die alltägliche Praxis professioneller Arbeit für und mit alten Menschen hohe Bedeutung haben. So erschienen Bände zu folgenden Themen: „Arzneimittel im Alter“, „Schmerz im Alter“, „Pflegebedürftigkeit im Alter“, „Ernährung im Alter“, „Demenzielle Erkrankungen im Alter“, „Mobilität und Verkehrssicherheit“, „Chronische Wunden im Alter“, „Zahn- und Mundgesundheit“ sowie „Migration und Alter“. Der vorliegende Band zum Thema „Gewalt und Alter“ vermittelt Wissen zur Thematik aus pflegewissenschaftlicher, medizinischer und rechtlicher Perspektive. Zudem werden Phänomene der Gewalt gegen ältere Menschen sowie Ansätze zur Prävention vorgestellt. Er bietet den Lesern einen aktuellen Überblick zum Thema „Gewalt und Alter“.

Die Reihe richtet sich traditionell an alle Berufsgruppen, die in gesundheitsrelevanten Versorgungsbereichen mit älteren und alten Menschen tätig sind. Der Band zum Thema Gewalt gegen alte Menschen kann aber darüber hinaus auch Betroffenen selbst und Angehörigen Informationen und Hilfestellung im Umgang mit diesem Thema bieten.

Als Initiatoren dieser Reihe und Bandherausgeber danken wir den Autorinnen und Autoren des Bandes für ihre spannenden Texte und sind zugleich Ralf Suhr zu Dank für die gute Zusammenarbeit verpflichtet. Frau Dr. Andrea Budnick danken wir für ihre kluge und stetige Unterstützung im Entstehungsprozess des Buches. Dem Verlag Walter De Gruyter sind wir weiterhin sehr dankbar, dass er unsere Idee zu dieser interdisziplinären Reihe umgesetzt hat.

In Dankbarkeit für die vielen Jahre gemeinsamer Arbeit schließen wir nun die Reihe „Praxiswissen Gerontologie und Geriatrie kompakt“ mit diesem zehnten Band.

Adelheid Kuhlmey und Wolfgang von Renteln-Kruse

https://doi.org/10.1515/9783110650341-201 
